\title{
Framework for Mobile based Expert System for Poultry Diseases
}

\author{
Abdelrahman Elsayed \\ Climate Change Information Center \& Renewable \\ Energy \& Expert Systems, \\ Agricultural Research Center \\ Giza, Egypt
}

\author{
Maryam Hazman \\ Climate Change Information Center \& Renewable \\ Energy \& Expert Systems, \\ Agricultural Research Center \\ Giza, Egypt
}

\begin{abstract}
This work presents a framework for the diagnosis and treatment of poultry diseases which is based on the expert system methodology. It aims to increase the availability of expert systems to run on mobile devices in offline mode to reach the area that has no internet connection. It will enable poultry breeders to diagnose and take proactive procedures to mitigate poultry diseases. According to the input symptoms, the app will diagnose poultry based on the suspect and confirm problem solver. A knowledge management module has been built to facilitate the knowledge acquisition process. So, we trained the domain expert to use it for entering their knowledge. The legacy inference engine was adapted to run on a mobile application. The presented framework was experiment in developing the chicken diagnosis and treatment disorders mobile application.
\end{abstract}

\section{General Terms}

Artificial Intelligence, Poultry diseases

\section{Keywords}

Expert systems, inference engine, Knowledge based systems, Mobile application

\section{INTRODUCTION}

As a result of the continuous increase in Egypt's population, developing and increasing agricultural productivity becomes an important issue to achieve food security. So, one of the main objectives of Sustainable Agricultural Strategy in Egypt is improving agricultural (land and animal) productivity [1]. Using information and communication technology to develop a system to assist poultry breeders in improving poultry productivity will contribute in that strategy objective. Expert systems technology provides an easy way to transfer the expertise of veterinary experts to the farmers (who is nonexpert). The Expert systems arose as a branch of applied artificial intelligence and were developed by the AI community in the mid-1960s [2]. The basic idea of developing expert systems is that human knowledge is captured in a computer program to solve the problems that usually require human expertise [3]. Then the computer can make inference and arrive to a specific conclusion [2]. The developing of the expert system idea is to implement the expertise knowledge into a computer program which can be consulted in the similar behavior with the human expert. A well-designed expert system simulates the reasoning process of the human expert to solve a particular problem. Therefore, non-expert people can use it to enhance their capabilities to solve problems. Moreover, experts can use it as knowledgeable assistants [4].

Poultry productivity is significantly affected by Poultry diseases. So, this work aims to develop a mobile app that helps farmers in protecting their poultries. The farmers will use this expert system app to diagnose and treat their farm's poultry diseases.

In this work, we introduce a framework for diagnosis expert system, which is based on CommonKADS knowledge engineering methodology [5]. In CommonKADS, the expert system includes three layers which are: domain knowledge, inference knowledge, and task knowledge [6]. The diagnosis problem is considered as a "suspect" and "confirm problem solver. Depending on the input symptoms, the problem solver method suspects some candidate diseases which is considered as suspected diseases. Then it tries to confirm one or more from these suspected diseases by asking the user about all symptoms which are related to the suspected disorders [7].

So in our expert system, the domain knowledge layer includes the poultry ontology for diagnosis and treatment, diagnosis model and treatment model. The model includes the relations between domain ontology items in a rule base representation. Inference knowledge layer comprises of the used inference steps needed in solving the problem, which are "suspect" and "confirm" for diagnosis and treat for treatment. While the task layer control the used inference in order to achieve expert system objective $[5,6]$. The proposed framework includes three main components: knowledge acquisition component, the expert system component, and mobile based interface. The knowledge acquisition component is used by the knowledge engineers and can be used by the veterinary experts to enter their expertise knowledge in the domain knowledge layer. While the mobile based interface is used by farmers to diagnosis and treat their farm poultries.

The presented framework was experiment in developing the chicken diagnosis and treatment disorders mobile application. This app was uploaded in Google play store; it can be downloaded for free.

The rest of this paper is organized as follows: Section 2 introduces the related work, section 3 introduces the problem statement, section 4 describes the proposed poultry diagnosis expert system framework, and section 5 presents the methodology. Section 6 is the evaluation. The final section presents our conclusion and future work.

\section{RELATED WORK}

Since the 1960s, many expert systems have been developed in different domains. One of those domains is animal disease diagnosis. SEDPA is an expert system for disease diagnosis in eel rearing systems [4]. It lowers the risk of getting a wrong decision and maximizes the possibility of making a right decision by means of a fuzzy controller and the DempsterShafer theory, so it can suggest several diseases with different belief levels, allowing the ultimate decision to be made by the 
manager of the fish farm [4]. DCDDS is a web-based expert system that mimics human expertise for diagnosing common dairy cow diseases. It includes a large amount of cow disease data and images which are used to conduct online disease diagnosis. It contains three models Case-based reasoning (CBR), Subjective Bayesian theory, and D-S evidential theory. According to the input group of symptoms, the system chooses which of the three models should be adopted to meet the best resolve [8]. Alarcón-Salvatierra and his colleagues proposed a rule-based expert system for cow disease diagnosis. It has two components: a rule-based engine and a knowledge base. Its knowledge base was represented using semantic web rule language. In order to diagnose a cow disease, the user enters a set of symptoms, and then the rulebased engine will identify the cow disease based on these symptoms [9].

As a result of the use and expansion of mobile technology, most mobile applications have been developed to help farmers in their daily duty. Diagnosis is one of these applications which were developed to help farmers diagnose animal and plant disorders. Suharjito and his colleagues introduced a mobile expert system for cows' reproduction health which used by farmers to diagnose their caw diseases. They applied the Fuzzy Tsukamoto method in order to diagnose endometritis disease in cows [10]. Also, Toseef and Khan used fuzzy logic as a decision-making engine in developing a mobile diagnosis expert system. Farmers use their system to diagnosis cotton and wheat disorders [11]. Nascimento and his colleges developed a mobile expert system for diagnosis insects. They introduced ENTOTECA which is used as an assistance tool in Brazil forest pests' management by both farmers and extension officers. Also, it can be used as a learning tool for forestry students [12]. Agustiono and his colleges presented a diagnostic mobile expert system which uses to integrate disease and pest diagnosis, treatment, and control for corn [13].

\section{PROBLEM STATEMENT}

As a result of spread of smartphone, farmers start using it for making calls and run agriculture applications. Also, they use smartphones as an alternative to traditional personal computers, Hence there is a need to empower farmers by availing agricultural expert systems to run on their smartphones. Furthermore, it is preferred to avail the application to run even there is a lack of internet connectivity in some regions. One of the most interesting activities of farmers is poultry production. Farmers invest in poultry breeding to enhance their income. On the other hand, poultry diseases may affect the productivities and causes loss of income, so this paper presents a mobile based expert system as a tool for helping farmers and new graduated veterinaries to diagnose poultry disease and providing them with the main proactive procedures to mitigate the effect of diseases.

From expert system point of view, knowledge acquisition is time consuming which is the main drawback of knowledge acquisition.

\section{THE PROPOSED FRAMEWORK}

The proposed framework aims to tackle the drawback of the knowledge acquisition process, so it introduced a knowledge acquisition component that enables the domain expert to enter the knowledge and modify it. Also, the framework has a mobile-based interface component to help farmers to install and run the expert systems in their mobiles.

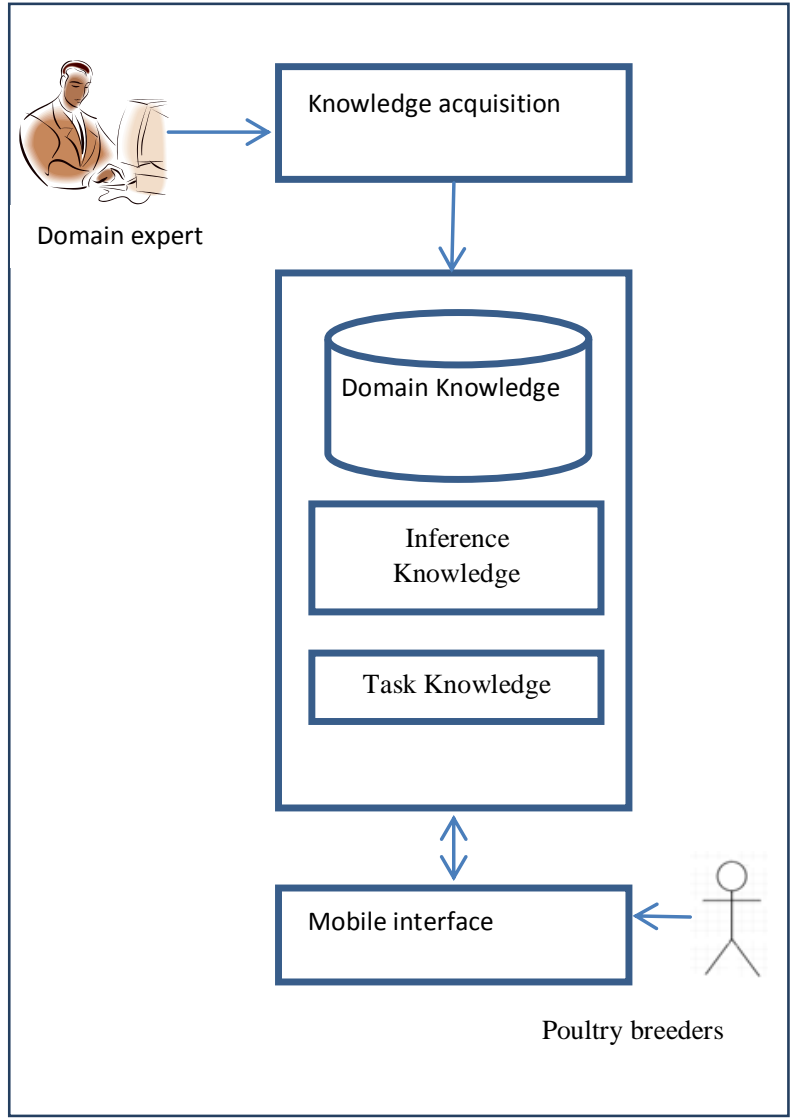

Fig 1: Mobile based poultry diagnosis and treatment application framework

Figure 1 displays the proposed framework. The heart of the proposed framework is the expert system component. It consists of domain knowledge, inference, and task layer. The Knowledge acquisition module is used by the domain expert in order to enter and review the domain knowledge which includes the disorders diagnosis rules, treatment rules, and diagnosis and treatment ontology. The poultry breeders interact with the proposed expert system via the mobile-based interface.

\section{METHODOLOGY}

To achieve our objective, we followed the following steps.

\subsection{Step1: acquire the domain knowledge}

Developing an expert system is similar to developing other software applications, but it requires several meetings with the domain expert in a specialized domain. We conducted several meetings with the domain experts to build the poultry ontology.

The poultry ontology includes all poultry concepts associated with its properties. Instead of defining concepts and their properties, we merge concepts and properties. For instance, we define concept property called mouth-shape instead of defining concept called mouth then defining property called shape. This approach facilitates the process of building the ontology and enables the domain expert to build the ontology by himself. Figure 2 displays the ontology editor. 


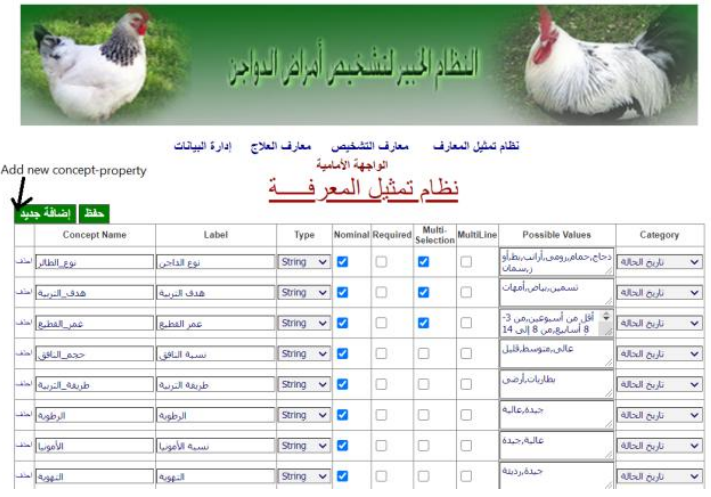

Fig 2: Ontology editor

Each concept-property has a set of attributes to describe it, for example, label, nominal, and required for search. In the case of concept-property is nominal we have to add the legal values of it, such as breeding objectives, Liver color, and the kidneys status.

The domain knowledge is represented as a set of rules. Each rule specifies a relation, recommendation, directive, strategy, or heuristic. A rule has the "if" part (which includes a set of conditions) and the "then" part (which includes a set of actions). In our diagnosis model, the disease name is the action of the rule which includes one action for simplicity to the domain expert. While the rule conditions represent the disease's symptoms. Each disease can have one rule or more rules. Figure 3 shows a rule in the diagnostic poultry model. Also, each disease can have one rule or more rules in the treatment module. The condition part has the disease name and the conclusion has the treatment needed for diagnosis of this disease.

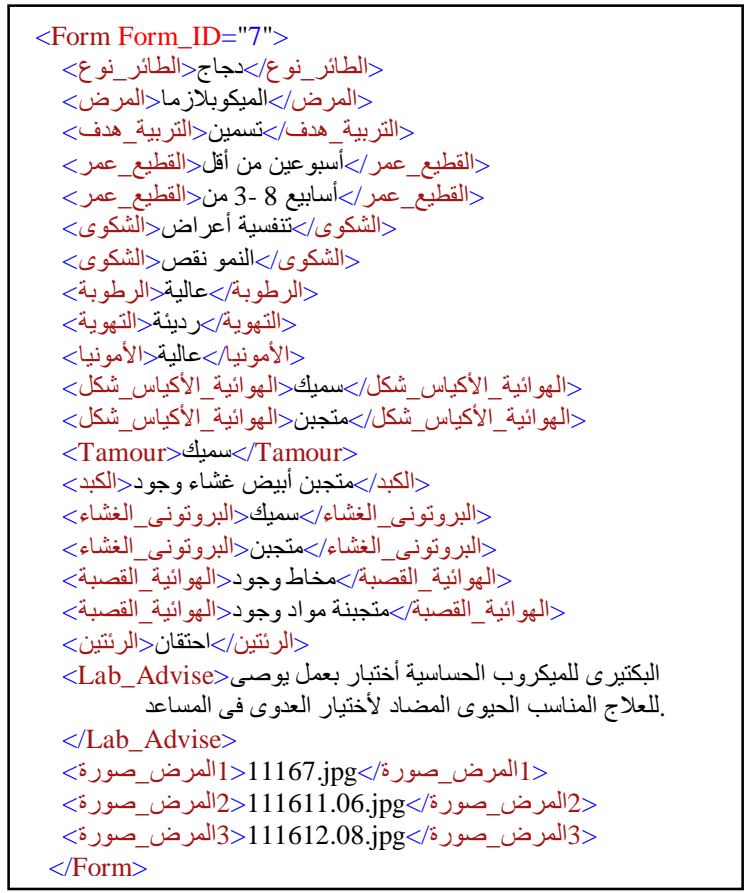

\section{Fig 3: Diagnostic rule in poultry expert system}

To facilitate the knowledge acquisition process we built a knowledge acquisition module. So, we trained the domain expert to use it for entering their knowledge. For each disease, the domain expert adds disease external and anatomical symptoms. If there any symptoms not exist in the ontology, the domain expert can add them to the ontology, hence he can continue adding disease rules.

\subsection{Step2: adapt the inference engine for diagnosis problem}

The domain expert has to specify the set of concept-properties that will be used in the suspect inference step, to differentiate between suspect symptoms and confirm symptoms. The specified suspect concepts are "poultry species, breeding objective, age, and main complaint).

The suspect inference step responsibility is to detect the suspected diseases according to some main symptoms. For example, when the breeding objective is fattening and chicken age is from (3 to 8 ) weeks and the value of the symptom "main complaint" is respiratory symptoms, it will suspect a subset of the poultry diseases (Newcastle, Infectious bronchitis IB, flu, Mycoplasma, Coli microbe). Indeed, the inference will narrow the search to 5 diseases instead of all diseases.

Figure 4 shows the suspect step of the diagnosis Inference engine algorithm. According to the suspect result, the confirm inference step starts asking the user about the symptoms which are related to the suspected diseases, so the system will iterate over all these symptoms. Based on the answer of the user the expected diseases list will be modified.

The treatment problem solver contains one domain model which is "Treatment". Each rule associates with the disease its treatment, proactive procedures, and pest control.

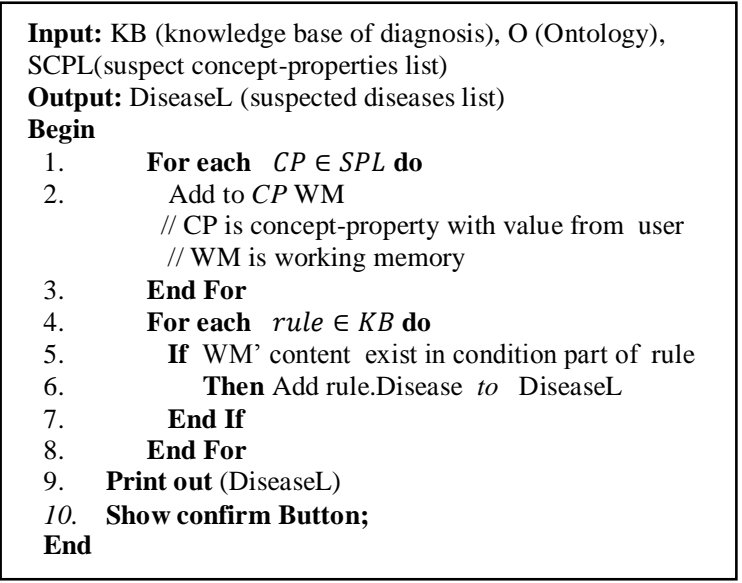

Fig 4: Suspect step algorithm

\subsection{Step3: generate mobile based interface}

After finishing the knowledge acquisition process we get XML files of the knowledge base (poultry ontology, diagnosis, and treatment). We used the Android Studio to build the poultry mobile application. Further, we used JavaScript integrated with XPath to parse the XML and building the inference. The mobile app runs offline without an internet connection, so the farmer can use it even if there is no internet connection.

\section{EVALUATION}

The proposed framework was evaluated by developing a mobile app for poultry diagnosis and treatment expert system. We selected chicken as a common type of poultry in the Egyptian countryside. The ontology includes about 42 concept-properties that cover chicken diseases. When the enduser runs the diagnosis sub-system, the expert system asks for poultry species, the purpose of breeding, age, and the main 
complaint as shown in figure 5.

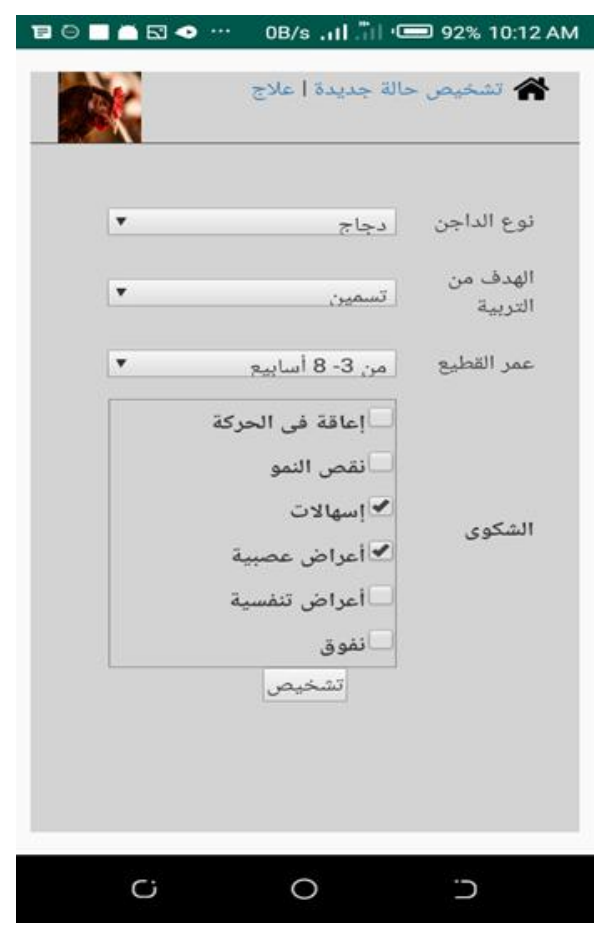

Fig 5: Suspected poultry symptoms interface

Supposing the end-user provides initial data such as chicken category is "دجاج", the purpose of breeding is "تسمين", and age is "من 8-3 اسابيع". The inference will generate and display the complaint's legal value which related to the input data values. After selecting the complaint value, the farmer has to click on diagnosis ("تشخيص"). Then, the app will display the diseases which are returned by the suspected inference as shown in figure 6.

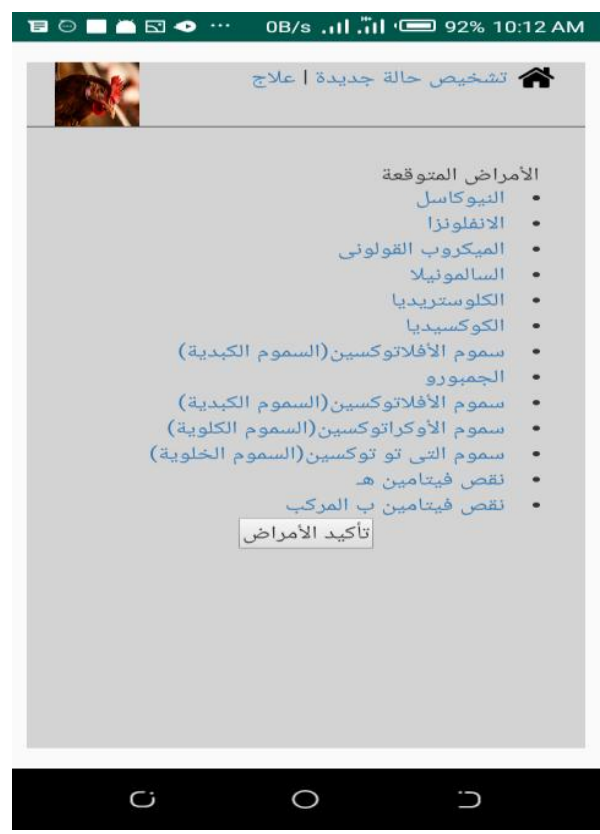

Fig 6: The suspected disorders list

The end-user can view the cases of the disease by clicking the disease link. In order to complete diagnostic, the farmer will click confirm disorders "تتأكيد الامر اض". The confirm inference engine will use the suspected disease rules to continue the diagnosis task. The dialogue will start between the mobile app and the user. The app will ask its user the set of questions that are generated from the suspected disease rules till it reaches a confirmed disorder(s). Figure 7 displays the confirmation phase of the diagnosis subsystem.

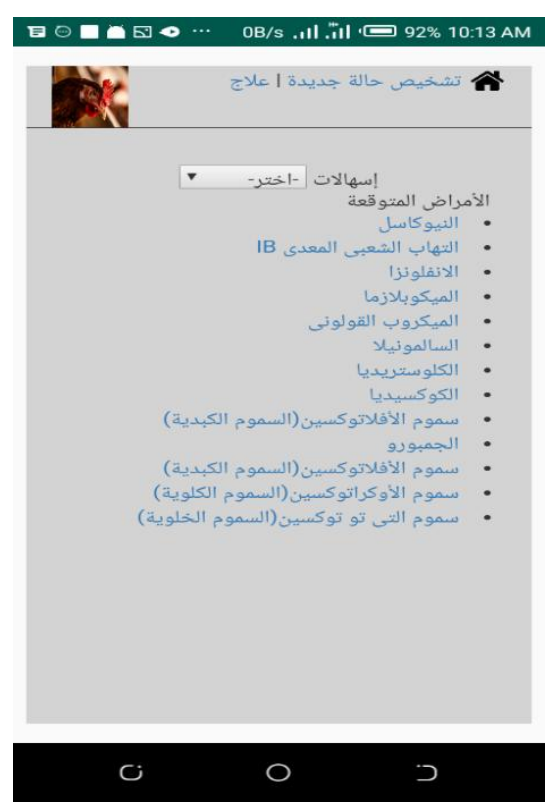

Fig 7: The confirmation phase for suspected disorders

The end-user will use the treatment sub-system for treatment and proactive procedures to control poultry detected disorders. First, the end-user selects the disease, after that the treatment inference engine will fire the treatment model to display the steps of pest control, treatment, or one of them based on the selected disease. As shown in figure 8 the system displays proactive procedures and how to control avian flu disease.

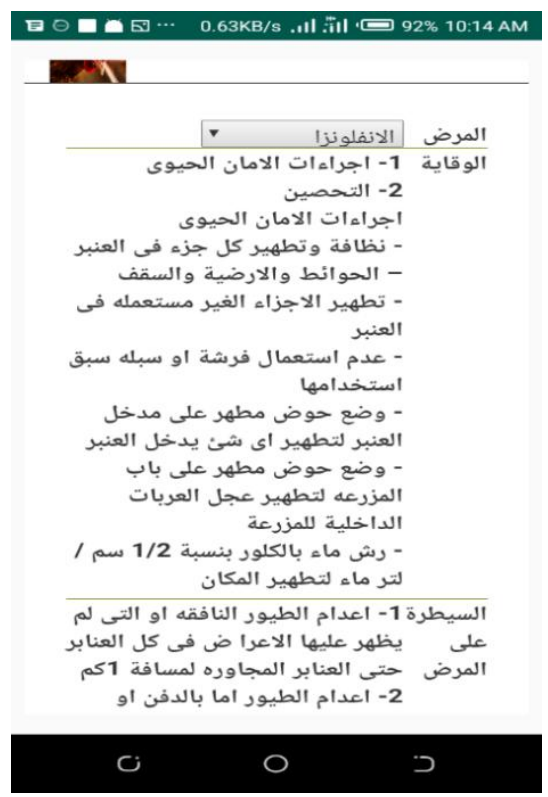

Fig 8: The treatment interface

\subsection{Poultry Diagnosis Expert System Ratings}

The Application quality has been reviewed and assessed by 24 users, each user gives a score from (1 to 5 stars) for the 
recommendation of the application. Table 1 displays the rating results. The average score was 4.3 , which means that the application has been reached excellent satisfaction.

Table 1, Ratings of poultry diagnosis application

\begin{tabular}{|l|l|l|l|l|l|}
\hline Rate & $* * * * *$ & $* * * *$ & $* * *$ & $* *$ & $*$ \\
\hline \# users & 17 & 2 & 3 & 0 & 2 \\
\hline
\end{tabular}

\section{CONCLUSION AND FUTURE WORK}

Poultry production is an important field in economics. Controlling disorder increase poultry production and satisfy food security. So, this paper introduced a framework for developing a mobile application for diagnosing and treatment poultry disorders. The framework composes of three main components: knowledge acquisition, the expert system, and mobile based interface.

The poultry mobile application is a useful tool for assisting farmers in diagnosing and treating poultry diseases and tool for sharing knowledge. The framework was evaluated by developing a chicken diagnosis and treatment application. The mobile application was developed on an Android platform, the poultry knowledge base was represented as XML files. The knowledge was acquired from the veterinary expert using the knowledge acquisition component. It runs in offline mode, so the knowledge will be available even if there is a lack of internet connectivity.

For future work, we need to implement expert system for poultry care and feeding. Also, we will integrate the symptoms with images that can be used instead of text legal value. Using the input as a natural language is one of the future work which we will investigate in it.

\section{REFERENCES}

[1] MALR 2009. Sustainable Agricultural Development Strategy towards 2030 Cairo, Ministry of Agriculture and Land Reclamation, Egypt, cairo.

[2] Karagiannis, S., Dounis, A.I., Chalastras,T., Tiropanis, P. and Papachristos, D., 2007. Design of expert system for search allergy and selection of the skin tests using CLIPS. International Journal of Biomedical and Biological Engineering, 1(7), pp.461-464.

[3] Diana-Aderina, M. and Anca-Petruţa, N., 2011. The Use Of Expert Systems In Agricultural Insurance. Necessity Vs. Reality. Annals of Faculty of Economics, University of Oradea-Faculty of Economics, pp.396-401.
[4] Gutierrez-Estrada, J.C., Sanz, E.D.P., López-Luque, R. and Pulido-Calvo, I., 2005. SEDPA, an expert system for disease diagnosis in eel rearing systems. Aquacultural engineering, 33(2), pp.110-125.

[5] Breuker, J. and Van de Velde, W. eds., 1994 CommonKADS library for expertise modelling: reusable problem solving components (Vol. 21). IOS press.

[6] Schreiber, G., Wielinga, B., de Hoog, R., Akkermans, H. and Van de Velde, W., 1994. CommonKADS: A comprehensive methodology for KBS development. IEEE expert, 9(6), pp.28-37.

[7] Moawad, N. and Elsayed, A., 2020, November. Smartphone Application for Diagnosing Maize Diseases in Egypt. In 2020 14th International Conference on Innovations in Information Technology (IIT) (pp. 24-28). IEEE.

[8] Rong, L. and Li, D., 2007, August. A web based expert system for milch cow disease diagnosis system in China In International Conference on Computer and Computing Technologies in Agriculture (pp. 1441-1445). Springer, Boston, MA.

[9] Alarcón-Salvatierra, A., Bazán-Vera, W., EspinozaMoran, W., Arcos-Jácome, D. and Burgos-Herreria, T., 2019, January. A Rule-Based Expert System for Cow Disease Diagnosis. In 2nd International Conference on ICTs in Agronomy and Environment (pp. 29-37). Springer, Cham.

[10] Suharjito, Diana, Yulyanto, and Nugroho, A., Mobile expert system using fuzzy tsukamoto for diagnosing cattle disease. Procedia computer science, 116, pp.27-36.

[11] Toseef, M. and Khan, M., 2018. An intelligent mobile application for diagnosis of crop diseases in Pakistan using fuzzy inference system. Computers and Electronics in Agriculture, 153, pp.1-11

[12] Nascimento, D., Anunciação, R., Arnhold, A., Ferraz Filho, A., dos Santos, A. and Zanuncio, J., 2016. Expert system for identification of economically important insect pests in commercial teak plantations. Computers and Electronics in Agriculture, 121, pp.368-373.

[13] Agustiono, W., Syarief, M., Anamisa, D., Damayanti, F. and Yusuf, M., 2020, July. A Conceptual Model of Mobile Expert System for Integrated Pest and Disease Control: The Case of MyCorn. In Journal of Physics: Conference Series (Vol. 1569, No. 2, p. 022057). IOP Publishing. 